\title{
Theoretical Investigation on the Solubilization in Water of Functionalized Single-Wall Carbon Nanotubes
}

\author{
Michael Mananghaya, ${ }^{1,2}$ Emmanuel Rodulfo, ${ }^{1}$ Gil Nonato Santos, ${ }^{1}$ and Al Rey Villagracia ${ }^{1}$ \\ ${ }^{1}$ Physics Department, De La Salle University-Manila, Taft Avenue, Manila 1004, Philippines \\ ${ }^{2}$ Physics Department, Mapúa Institute of Technology, Muralla Street, Intramuros, Manila 1002, Philippines
}

Correspondence should be addressed to Michael Mananghaya, mike_mananghaya@yahoo.com

Received 14 July 2011; Revised 28 August 2011; Accepted 5 September 2011

Academic Editor: Lifeng Dong

Copyright (C) 2012 Michael Mananghaya et al. This is an open access article distributed under the Creative Commons Attribution License, which permits unrestricted use, distribution, and reproduction in any medium, provided the original work is properly cited.

\begin{abstract}
An important technique to increase the solubility and reactivity of carbon nanotube is through functionalization. In this study, the effects of functionalization of some single-walled carbon nanotubes (SWCNTs) were investigated with the aid of density functional theory. The SWCNT model used in the study consists of a finite, $(5,0)$ zigzag nanotube segment containing $60 \mathrm{C}$ atoms with hydrogen atoms added to the dangling bonds of the perimeter carbons. There are three water-dispersible SWCNTs used in this study that were functionalized with (a) formic acid, as a model of carboxylic acid, (b) isophthalic acid, as a model aromatic dicarboxylic acid, and (c) benzenesulfonic acid, as a model aromatic sulfonic acid. Binding energies of the organic radicals to the nanotubes are calculated, as well as the HOMO-LUMO gaps and dipole moments of both nanotubes and functionalized nanotubes. Binding was found out to be thermodynamically favorable. The functionalization increases the electrical dipole moments and results in an enhancement in the solubility of the nanotubes in water manifested through favorable changes in the free energies of solvation. This should lower the toxicity of nanotubes and improve their biocompatibility.
\end{abstract}

\section{Introduction}

Single-walled carbon nanotubes (SWCNTs) are allotropes of carbon and possess various novel properties that make them useful in the field of nanotechnology and pharmaceuticals. SWCNTs are tubular in shape, made of graphite, nanometers in diameter, and several millimeters in length and have a very broad range of electronic, thermal, and structural properties. These properties vary with the kind of nanotubes defined by its diameter, length, chirality or twist, and wall nature. Their unique surface area, stiffness, strength, and resilience have led to much excitement in the field of pharmacy. Nanostructured materials hold promise for a wide range of technological applications and are increasingly studied, not only for their possible applications in electronics, optics and mechanical materials, but also specifically having enormous potential in nanomedicine [1-11]. Because of this, it is imperative to examine the toxicity of these carbon-based nanostructures. Previous toxicological evaluations of single-walled carbon nanotubes have been conducted, both in cell culture and in vivo. One example, using an SWCNT surfactant stabilized system where the Fe content was significantly high, reported an elevated cytotoxic response [12]. Warheit et al. [13] observed an increase in inflammatory response in the lung cavities of rats. While these studies report potential negative implications of SWCNT, they did not use a SWCNT sample easily dispersed in water via covalently bound functional groups.

Carbon nanotubes (CNTs) are insoluble in water. For successful biomedical applications of nanotubes the solubility problem may be overcome in part by chemical functionalization of the CNT surface, resulting in an increased compatibility of the functionalized CNT with water [1]. Functionalization has led to improved compatibility with a variety of biological components. Functionalization has been obtained both through non-covalent-binding schemes [10] or covalent binding at the CNT surface [7-9].

Studies done previously, evaluated the differential cytotoxicity of water-suspendable fullerenes on human dermal fibroblasts (HDFs) in culture [14]. It was concluded that as 


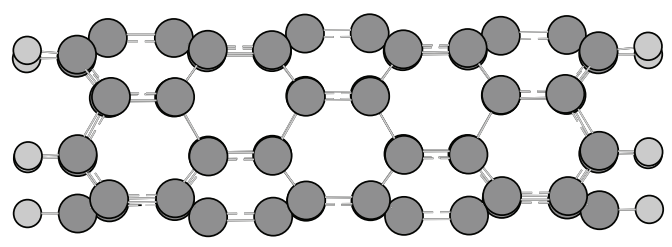

FIgURE 1: Optimized geometry of the finite $(5,0)$ zigzag SWCNT $\left(\mathrm{C}_{60} \mathrm{H}_{20}\right)$. Gray color depicts carbon atoms and light gray is hydrogen.

the degree of functionalization on the surface of the fullerene cage increases, the cytotoxicity of the fullerene decreases significantly. The simple act of functionalizing the fullerene with either carboxyl or hydroxyl groups decreased the cytotoxic response over seven orders of magnitudes for HDF, human liver carcinoma cells, and neuronal human astrocyte cell lines. Different molecules have been employed to functionalize CNT into customized species that may simultaneously carry several nanostructures (multifunctionalization) to detect, allow imaging; and deliver therapeutical loads [15].

Essentially, the need to improve biodistribution, pharmacokinetics, and solubility, as well as the need to diminish the toxicity of the CNT [1] are fundamental problems addressed through computational simulation and modeling. The aim of this research is to perform density functional theory (DFT) calculations [16] to investigate how the nanotube properties change due to covalent functionalization of certain organic groups to side walls and tips of the nanotubes, improving its solubility in water. Functionalization enhances the biocompatibility, potentially diminishing toxicity and therefore paves the way to rational modifications and/or design of new nanostructured species for efficient targeting and drug delivery.

\section{Methodology}

To investigate the effects of functionalization of SWCNT in increasing their solubility we build a finite model $(5,0)$ zigzag nanotube segment containing $60 \mathrm{C}$ atoms with hydrogen atoms added to the dangling bonds of the perimeter carbons and the resulting formula is $\mathrm{C}_{60} \mathrm{H}_{20}$, see Figure 1. This nanotube model system is similar to Jaffe's model [17]. The nanotubes are covalently functionalized with the following organic molecules: (a) formic acid, $\mathrm{CO}_{2} \mathrm{H}_{2}$, as a model of carboxylic acid, Figure 2 shows the functionalization at the sidewall and Figure 3 shows the functionalization at the tip of the SWCNT which is a typical product of the oxidation of carbon nanotubes $[9,10]$, (b) isophthalic acid, $\mathrm{C}_{6} \mathrm{H}_{4}\left(\mathrm{CO}_{2} \mathrm{H}\right)_{2}$, as a model aromatic dicarboxylic acid, see Figure 4, [18], and (c) benzenesulfonic acid, $\mathrm{C}_{6} \mathrm{H}_{5} \mathrm{SO}_{3} \mathrm{H}$, as a model aromatic sulfonic acid, see Figure 5 [18]. These molecules have been investigated for their capability of drug delivery and diagnostic applications.

All calculations were performed using DFT with a hybrid functional B3LYP [19-22] and a 3-21 G basis set [23]. Charge densities were analyzed by the Mulliken method [24]. For open-shell molecular radicals, the unrestricted

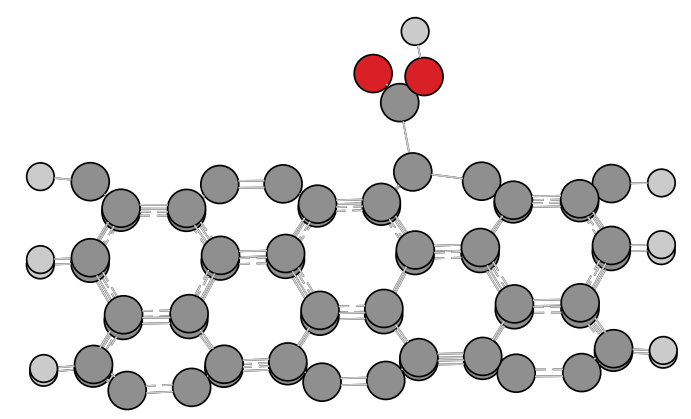

Figure 2: Optimized geometry of the finite $(5,0)$ zigzag SWCNT $\left(\mathrm{C}_{60} \mathrm{H}_{20}\right)$ with the formic acid radical at the tube sidewall. Gray color depicts carbon atoms; light gray is hydrogen and red is oxygen.

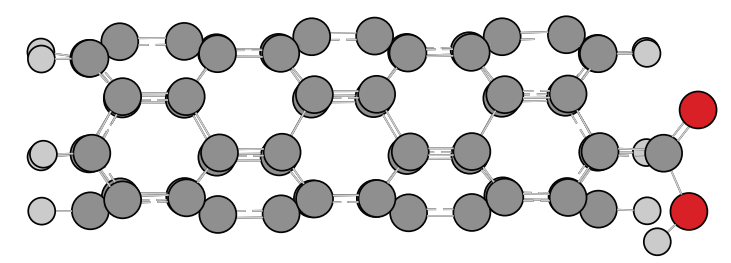

Figure 3: Optimized geometry of the finite $(5,0)$ zigzag SWCNT $\left(\mathrm{C}_{60} \mathrm{H}_{19}\right)$ with the formic acid radical at the tube tip. Gray color depicts carbon atoms; light gray is hydrogen and red is oxygen.

formalism was used. The present level of calculation, DFT (UB3LYP)/3-21 G, is known to produce reasonable results [25] for bond lengths, bond angles, and bond energies for a wide range of molecules. The computations were carried out on a computer using the $a b$ initio quantum chemistry package, Gaussian 09 (G09) [26], with the default convergence thresholds for DFT optimization calculations.

Electronic structure descriptors have been computed to analyze the geometrical and electronic changes that may lead to a better solubility of the functionalized nanotubes. Among them are the electronic HOMO-LUMO gap (H-L gap), the dipole moment $\left(\mu_{\text {dip }}\right)$, and the Gibbs free energy of solvation $\left(\Delta \mathrm{G}_{\text {solv }}\right)$. The HOMO-LUMO gap, in the case of open electronic shell systems, is computed between the highest of the HOMOs and the lowest of the LUMOs, regardless of the spin type. For all systems with a doublet multiplicity (one unpaired electron), this always resulted in the HOMO (alpha)-LUMO (beta) gap.

The solubility of a given molecule in a solvent can be assessed by measuring its Gibbs free energy of solvation. A number of quantum mechanical continuum solvation models were developed for this purpose [27, 28]. We have chosen the polarizable continuum model (PCM) as implemented in G09. This originated from the Onsager continuum model [28] and was formulated by Tomasi et al. [27, 29-31], which takes into account the solute interactions with the solvent, modeled as a continuum dielectric medium in which a cavity is built to accommodate the solute molecule.

\section{Results and Discussions}

3.1. Structural Parameters. We have used the G09 code to optimize the geometries for the finite SWCNT. The resulting 


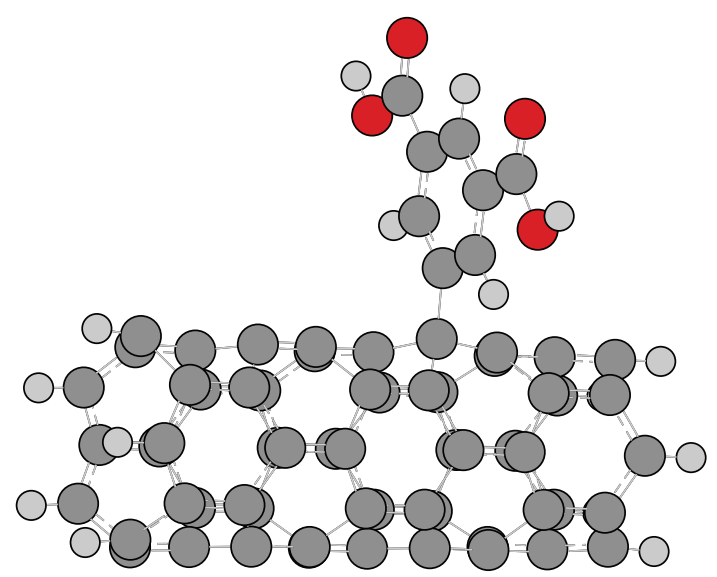

FIgure 4: Optimized geometry of the finite $(5,0)$ zigzag SWCNT $\left(\mathrm{C}_{60} \mathrm{H}_{20}\right)$ with the isophthalic acid radical at the tube sidewall. Gray color depicts carbon atoms; light gray is hydrogen and red is oxygen.

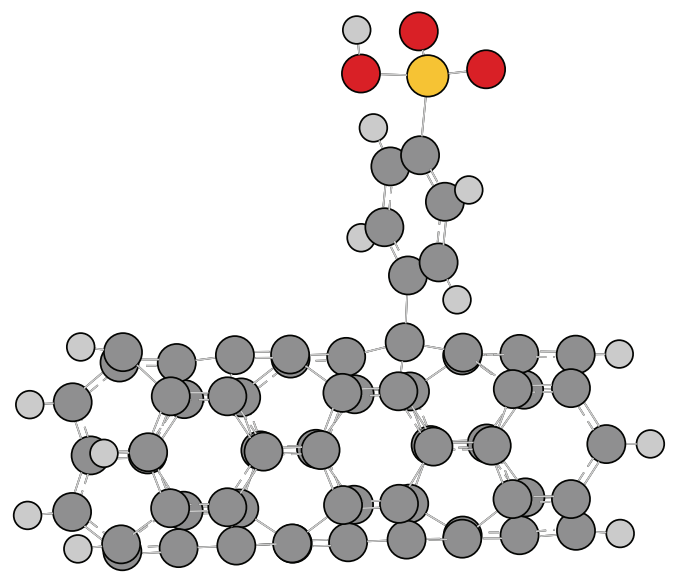

FIGURE 5: Optimized geometry of the finite $(5,0)$ zigzag SWCNT $\left(\mathrm{C}_{60} \mathrm{H}_{20}\right)$ with the benzenesulfonic acid radical at the tube sidewall. Gray color depicts carbon atoms; light gray is hydrogen, yellow is sulfur and red is oxygen.

optimized structures are shown in Figure 1 . The finite $(5,0)$ nanotube $\mathrm{C}_{60} \mathrm{H}_{20}$ has an optimized length of $11.32 \AA$ and a diameter of $3.90 \AA$. Computed length agrees with a value of $11.36 \AA$ obtained by Jaffe's model [17] and computed SWCNT diameter also compares well with the value $3.92 \AA$ in their paper.

Presented in Figures 2 and 3 are the optimized structures for two possible ways of functionalizing the finite SWCNT with formic acid. Figure 2 shows the functionalization at the sidewall of the nanotube wherein we take the organic molecule already in its radical form, $\mathrm{CO}_{2} \mathrm{H}$, attached by its $\mathrm{C}$ atom to the nanotube sidewall. In Figure 3 the functionalization at the tip of the SWCNT was carried out as another possibility because it is known that closed nanotube tips react with strong oxidizing agents, opening the tip and filling the dangling bonds with carboxylic acid molecules. The optimized structure for the functionalization of the SWCNT sidewall with the isophthalic acid radical ${ }^{\circ} \mathrm{C}_{6} \mathrm{H}_{3}\left(\mathrm{CO}_{2} \mathrm{H}\right)_{2}$. is given in Figure 4. We have also studied the functionalization of the SWCNT sidewall with the benzenesulfonic acid, $\mathrm{C}_{6} \mathrm{H}_{4} \mathrm{SO}_{3} \mathrm{H}$; the optimized structure is shown in Figure 5. These molecules have been used in studies of covalent functionalization of SWCNT capability for drug delivery $[15,18]$.

3.2. Binding Energies. Binding energies of the radicals to the nanotube, $\mathrm{E}_{\mathrm{b}}$, are defined for the reaction

$$
\text { ORG + SWCNT } \longrightarrow \text { ORG-SWCNT, }
$$

where ORG is any of the studied organic radicals and ORGSWCNT is the corresponding functionalized nanotube. Therefore

$$
\mathrm{E}_{\mathrm{b}}=\mathrm{E}(\mathrm{ORG}-\mathrm{SWCNT})-\mathrm{E}(\mathrm{SWCNT})-\mathrm{E}(\mathrm{ORG}) .
$$

The binding energy is negative if functionalization is thermodynamically favorable. To avoid considering the artificial rupture of $\mathrm{C}-\mathrm{H}$ bonds involving functionalization at the tip, one $\mathrm{H}$ atom was previously removed and the nanotube structure was reoptimized before functionalization with the formic acid radical. The studied reactions are:

$$
\begin{aligned}
& \mathrm{CO}_{2} \mathrm{H}+\mathrm{C}_{60} \mathrm{H}_{20} \\
& \quad \longrightarrow \mathrm{CO}_{2} \mathrm{H}-\mathrm{SWCNT} \quad \text { (on sidewall) } \\
& \mathrm{C}_{6} \mathrm{H}_{3}\left(\mathrm{CO}_{2} \mathrm{H}\right)_{2}+\mathrm{C}_{60} \mathrm{H}_{20} \\
& \quad \longrightarrow \mathrm{C}_{6} \mathrm{H}_{3}\left(\mathrm{CO}_{2} \mathrm{H}\right)_{2} \text {-SWCNT (on sidewall) } \\
& \mathrm{C}_{6} \mathrm{H}_{4} \mathrm{SO}_{3} \mathrm{H}+\mathrm{C}_{60} \mathrm{H}_{20} \\
& \quad \longrightarrow \mathrm{C}_{6} \mathrm{H}_{4} \mathrm{SO}_{3} \mathrm{H}-\mathrm{SWCNT} \quad \text { (on sidewall) } \\
& \mathrm{CO}_{2} \mathrm{H}+\mathrm{C}_{60} \mathrm{H}_{19} \\
& \quad \longrightarrow \mathrm{CO}_{2} \mathrm{H}-\mathrm{SWCNT} \quad \text { (on tip) }
\end{aligned}
$$

Since the energies of the organic radicals are required in (2), their structures were also optimized. The functionalization reactions (3) to (6) are thermodynamically favorable, as can be seen in Table 1, and the resulting binding energies are between $-2 \mathrm{eV}$ to $-4 \mathrm{eV}$.

3.3. Electronic Properties. Electronic properties computed for the functionalized SWCNT is displayed in Table 1 for the $\mathrm{C}_{60} \mathrm{H}_{20}$ nanotube and the functionalized SWCNT. These include the HOMO-LUMO gaps (H-L gap), the dipole moments $\left(\mu_{\text {dip }}\right)$; and the Gibbs free energy of solvation $\left(\Delta \mathrm{G}_{\text {solv }}\right)$. The HOMO-LUMO gap of the infinite $(5,0)$ SWCNT is zero, because that nanotube is metallic. A negligible HOMO-LUMO gap develops in the finite nanotube model $(0.05 \mathrm{eV})$, in agreement. The spatial distributions of the HOMO orbitals of the SWCNT and the tube with $\mathrm{H}$ atom missing from the tip, SWCNT radical, are shown in Figure 6. These orbitals display a similar delocalization over the whole tube, but the main difference is that the HOMO of the SWCNT radical has a noticeable lobe at the defect, suggesting a dangling bond at this site. Spatial 
TABle 1: Electronic properties for the $\mathrm{C}_{60} \mathrm{H}_{20}$ nanotube and the functionalized SWCNT formed in the reactions (3)-(6). The properties are HOMO-LUMO gap (H-L gap), magnitude of the dipole moment $\left(\mu_{\mathrm{dip}}\right)$, and the Gibbs free energy of solvation $\left(\Delta \mathrm{G}_{\text {solv }}\right)$.

\begin{tabular}{|c|c|c|c|c|}
\hline System & $\mathrm{E}_{\mathrm{b}}(\mathrm{eV})$ & $\begin{array}{c}\text { H-L gap } \\
(\mathrm{eV})\end{array}$ & $\begin{array}{c}\mu_{\text {dip }} \\
\text { (debye) }\end{array}$ & $\begin{array}{c}\Delta \mathrm{G}_{\text {solv }} \\
(\mathrm{eV})\end{array}$ \\
\hline SWCNT & - & 0.05 & 0.34 & -0.73 \\
\hline $\begin{array}{l}\mathrm{CO}_{2} \mathrm{H}-\mathrm{SWCNT} \\
\text { (sidewall) }\end{array}$ & -2.00 & 0.38 & 3.60 & -0.98 \\
\hline $\begin{array}{l}\mathrm{C}_{6} \mathrm{H}_{3}\left(\mathrm{CO}_{2} \mathrm{H}\right)_{2}- \\
\text { SWCNT } \\
\text { (sidewall) }\end{array}$ & -2.73 & 0.41 & 3.96 & -1.16 \\
\hline $\begin{array}{l}\mathrm{C}_{6} \mathrm{H}_{4} \mathrm{SO}_{3} \mathrm{H}- \\
\text { SWCNT } \\
\text { (sidewall) }\end{array}$ & -3.59 & 0.38 & 3.80 & -1.12 \\
\hline $\begin{array}{l}\mathrm{CO}_{2} \mathrm{H}-\mathrm{SWCNT} \\
\text { radical (tip) }\end{array}$ & -3.88 & 0.41 & 9.79 & -1.05 \\
\hline
\end{tabular}

distributions of the LUMO orbitals for the same nanotubes are given in Figure 7. A prominent accumulation of the orbital distribution appears at the vacancy position of the missing $\mathrm{H}$ atom in the SWCNT radical. The localized lobes are responsible for the reactivity of the nanotube.

In Table 1 we can observe an important increase of the magnitude of the dipole moments of the functionalized nanotubes compared to the nanotube that is not functionalized. The $\mathrm{C}_{60} \mathrm{H}_{20}$ has a small dipole moment of 0.34 debye and the values of the dipole moments of the functionalized SWCNT are much larger. The substantial increase in the dipole moment is expected to modify the interaction of the functionalized nanotubes with a polar solvent such as water, potentially increasing their solubilities in the physiological medium.

3.4. Solubilization. Solubility can be assessed by measuring its Gibbs free energy of solvation. The Gibbs free energy of solvation, $\Delta \mathrm{G}_{\text {solv }}$, is computed as the difference between the optimized energies of the species in the solvent (PCM) and vacuum (gas phase),

$$
\Delta \mathrm{G}_{\text {solv }}=\mathrm{E}_{\mathrm{PCM}}-\mathrm{E}_{\text {gas }} .
$$

The PCM; as implemented in G09 which takes into account the solvent interactions with the solute within a cavity in a continuum dielectric medium approximation for the solvent; was used to calculate the optimized energies of the species in the solvent such as water. Solubility of a given molecule in water requires a negative value of $\Delta \mathrm{G}_{\text {solv }}$. All the calculated $\Delta \mathrm{G}_{\text {solv }}$ of the functionalized nanotubes, given in Table 1, are negative. If we compare with the $\Delta \mathrm{G}_{\text {solv }}$ obtained for the unfunctionalized nanotube, we see that solubility in water is larger for the functionalized nanotubes. This is in agreement with the experiment performed in vitro cytotoxicity screens on HDF by Sayes et al. [18] wherein the sidewall functionalized SWCNT with phenyl- $\mathrm{SO}_{3} \mathrm{H}$ and phenyl- $(\mathrm{COOH})_{2}$ samples are found out to be substantially less cytotoxic than unfunctionalized SWCNT in water. On

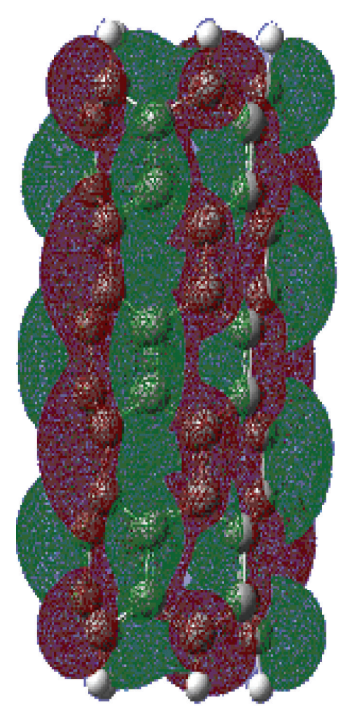

(a)

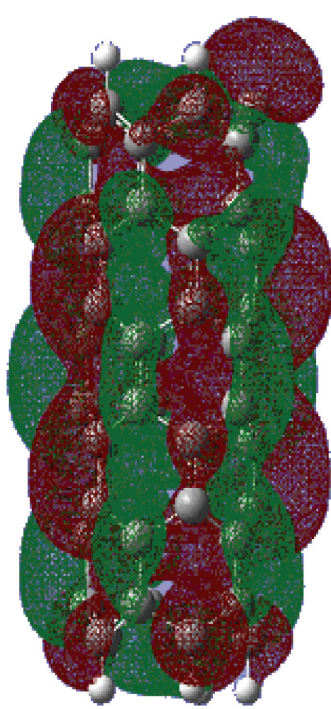

(b)
FIGURE 6: Spatial distribution of the HOMO orbitals for (a) the $\mathrm{C}_{60} \mathrm{H}_{20}$ nanotube and (b) the nanotube radical with a missing $\mathrm{H}$ atom from the tip $\mathrm{C}_{60} \mathrm{H}_{19}$. The wave function isosurfaces of values +0.01 and $-0.01\left(\mathrm{e} / \AA^{3}\right)^{1 / 2}$ are shown in red and green, respectively.

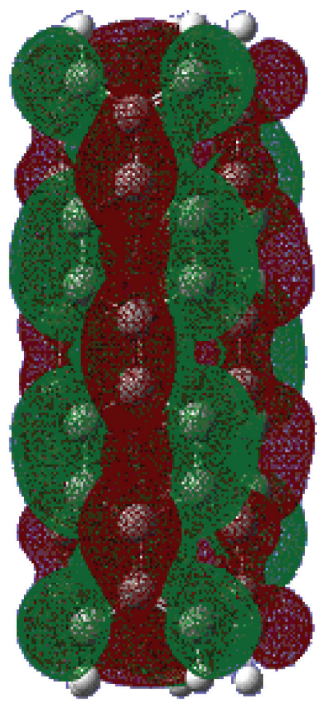

(a)

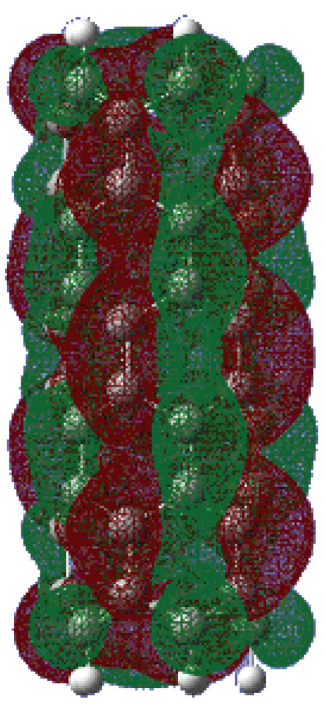

(b)
FIGURE 7: Spatial distribution of the LUMO orbitals for (a) the $\mathrm{C}_{60} \mathrm{H}_{20}$ nanotube and (b) the nanotube radical with a missing $\mathrm{H}$ atom from the tip $\mathrm{C}_{60} \mathrm{H}_{19}$. The wave function isosurfaces of values +0.01 and $-0.01\left(\mathrm{e} / \AA^{3}\right)^{1 / 2}$ are shown in red and green, respectively.

the other hand, the solvation Gibbs free energy for the SWCNT is $-0.73 \mathrm{eV}$, which is predicted to be slightly soluble. It is a little surprising for the tube, but this is an artifact of the finite model with artificial $\mathrm{C}-\mathrm{H}$ bonds at the tips. These slightly polarizable bonds induce an artificial minor solubility in the model SWCNT. However, in all cases, the solubility increases dramatically and substantially by using the technique of functionalization with the said organic radicals. 


\section{Conclusions}

Density functional theory using mainly the G09 code was used to investigate the functionalization of SWCNT with open ends capped with $\mathrm{H}$ atoms with three different kinds of acids that help to improve their solubility in water and could make nanotubes more biocompatible. The nanotube has been functionalized with formic acid, isophthalic acid; and benzenesulfonic acid. Electronic exchange and correlation effects have been treated with the gradient-corrected B3LYP functional.

The functionalization with these organic molecules, either on the nanotube sidewall or tip sites where a hydrogen atom has been removed, is thermodynamically favorable. The enhanced dipole moment of the nanotubes due to functionalization is manifested in the improvement of their solubility in water as assessed by a calculation of the solvation free energies of the functionalized nanotubes employing the PCM polarizable continuum model. Functionalization enhances nanotubes biocompatibility, thus reducing the toxicity and the chance of tissue accumulation, for their future use in drug delivery.

\section{Acknowledgments}

This work was supported in part by the Department of Science and Technology, Philippine Council for Advanced Science and Technology Research and Development (DOST, PCASTRD), Philippine Council for Industry, Energy and Emerging Technology Research and Development (PCIEERD) and by the De La Salle University-Manila Faculty. The ideas in this paper would not have crystallized in the present form without the incisive remarks of Melanie Y. David.

\section{References}

[1] N. Sinha and J. T. W. Yeow, "Carbon nanotubes for biomedical applications," IEEE Transactions on Nanobioscience, vol. 4, no. 2, pp. 180-195, 2005.

[2] N. Sinha, J. Ma, and J. T. W. Yeow, "Carbon nanotube-based sensors," Journal of Nanoscience and Nanotechnology, vol. 6, no. 3, pp. 573-590, 2006.

[3] C. Klumpp, K. Kostarelos, M. Prato, and A. Bianco, "Functionalized carbon nanotubes as emerging nanovectors for the delivery of therapeutics," Biochimica et Biophysica Acta, vol. 1758, no. 3, pp. 404-412, 2006.

[4] A. Bianco, K. Kostarelos, and M. Prato, "Applications of carbon nanotubes in drug delivery," Current Opinion in Chemical Biology, vol. 9, no. 6, pp. 674-679, 2005.

[5] A. Bianco, "Carbon nanotubes for the delivery of therapeutic molecules," Expert Opinion on Drug Delivery, vol. 1, no. 1, pp. 57-65, 2004.

[6] P. Couvreur and C. Vauthier, "Nanotechnology: intelligent design to treat complex disease," Pharmaceutical Research, vol. 23, no. 7, pp. 1417-1450, 2006.

[7] R. Singh, D. Pantarotto, D. McCarthy et al., "Binding and condensation of plasmid DNA onto functionalized carbon nanotubes: toward the construction of nanotube-based gene delivery vectors," Journal of the American Chemical Society, vol. 127, no. 12, pp. 4388-4396, 2005.

[8] A. Bianco, K. Kostarelos, C. D. Partidos, and M. Prato, "Biomedical applications of functionalised carbon nanotubes," Chemical Communications, no. 5, pp. 571-577, 2005.

[9] M. Prato, K. Kostarelos, and A. Bianco, "Functionalized carbon nanotubes in drug design and discovery," Accounts of Chemical Research, vol. 41, no. 1, pp. 60-68, 2008.

[10] N. W. S. Kam and H. Dai, "Carbon nanotubes as intracellular protein transporters: generality and biological functionality," Journal of the American Chemical Society, vol. 127, no. 16, pp. 6021-6026, 2005.

[11] A. De La Zerda, C. Zavaleta, S. Keren et al., "Carbon nanotubes as photoacoustic molecular imaging agents in living mice," Nature Nanotechnology, vol. 3, no. 9, pp. 557-562, 2008.

[12] N. W. S. Kam, T. C. Jessop, P. A. Wender, and H. Dai, "Nanotube molecular transporters: internalization of carbon nanotube-protein conjugates into mammalian cells," Journal of the American Chemical Society, vol. 126, no. 22, pp. 68506851, 2004.

[13] D. B. Warheit, B. R. Laurence, K. L. Reed, D. H. Roach, G. A. M. Reynolds, and T. R. Webb, "Comparative pulmonary toxicity assessment of single-wall carbon nanotubes in rats," Toxicological Sciences, vol. 77, no. 1, pp. 117-125, 2004.

[14] C. M. Sayes, J. D. Fortner, W. Guo et al., "The differential cytotoxicity of water-soluble fullerenes," Nano Letters, vol. 4, no. 10, pp. 1881-1887, 2004.

[15] X. Yang, Z. Zhang, Z. Liu, Y. Ma, R. Yang, and Y. Chen, "Multifunctionalized single-walled carbon nanotubes as tumor cell targeting biological transporters ," Journal of Nanoparticle Research, vol. 10, no. 5, pp. 815-822, 2008.

[16] R. G. Parr and W. Yang, Density Functional Theory of Atoms and Molecules, Oxford University Press, Oxford, UK, 1989.

[17] R. L. Jaffe, "Quantum chemistry study of fullerene and carbon nanotube fluorination," Journal of Physical Chemistry B, vol. 107, no. 38, pp. 10378-10388, 2003.

[18] C. M. Sayes, F. Liang, J. L. Hudson et al., "Functionalization density dependence of single-walled carbon nanotubes cytotoxicity in vitro," Toxicology Letters, vol. 161, no. 2, pp. 135142, 2006.

[19] A. D. Becke, "Density-functional exchange-energy approximation with correct asymptotic behavior," Physical Review A, vol. 38, no. 6, pp. 3098-3100, 1988.

[20] C. Lee, W. Yang, and R. G. Parr, "Development of the ColleSalvetti correlation-energy formula into a functional of the electron density," Physical Review B, vol. 37, no. 2, pp. 785$789,1988$.

[21] S. H. Vosko, L. Wilk, and M. Nusair, "Accurate spin-dependent electron liquid correlation energies for local spin density calculations: a critical analysis," Canadian Journal of Physics, vol. 58, no. 8, pp. 1200-1211, 1980.

[22] A. D. Becke, "Density-functional thermochemistry. III. The role of exact exchange," The Journal of Chemical Physics, vol. 98, no. 7, pp. 5648-5652, 1993.

[23] J. S. Binkley, J. A. Pople, and W. J. Hehre, "Self-consistent molecular orbital methods. 21. Small split-valence basis sets for first-row elements," Journal of the American Chemical Society, vol. 102, no. 3, pp. 939-947, 1980.

[24] R. S. Mulliken, "Electronic population analysis on LCAO-MO molecular wave functions. I," The Journal of Chemical Physics, vol. 23, no. 10, pp. 1833-1840, 1955. 
[25] F. D’Souza, M. E. Zandler, P. M. Smith et al., "A ferroceneC60-dinitrobenzene triad: synthesis and computational, electrochemical, and photochemical studies," Journal of Physical Chemistry A, vol. 106, no. 4, pp. 649-656, 2002.

[26] M. J. Frisch, G. W. Trucks, H. B. Schlegel et al., Gaussian 09, Revision A.1, Gaussian, Inc., Pittsburgh, Pa, USA, 2009.

[27] J. Tomasi, B. Mennucci, and R. Cammi, "Quantum mechanical continuum solvation models," Chemical Reviews, vol. 105, no. 8, pp. 2999-3093, 2005.

[28] L. Onsager, "Electric moments of molecules in liquids," Journal of the American Chemical Society, vol. 58, no. 8, pp. 1486-1493, 1936.

[29] E. Cancès and B. Mennucci, "New applications of integral equations methods for solvation continuum models: ionic solutions and liquid crystals," Journal of Mathematical Chemistry, vol. 23, no. 3-4, pp. 309-326, 1998.

[30] E. Cancès, B. Mennucci, and J. Tomasi, "A new integral equation formalism for the polarizable continuum model: theoretical background and applications to Isotropic and anisotropic dielectrics," Journal of Chemical Physics, vol. 107, no. 8, pp. 3032-3041, 1997.

[31] B. Mennucci, E. Cancès, and J. Tomasi, "Evaluation of solvent effects in isotropic and anisotropic dielectrics and in ionic solutions with a unified integral equation method: theoretical bases, computational implementation, and numerical applications," Journal of Physical Chemistry B, vol. 101, no. 49, pp. 10506-10517, 1997. 

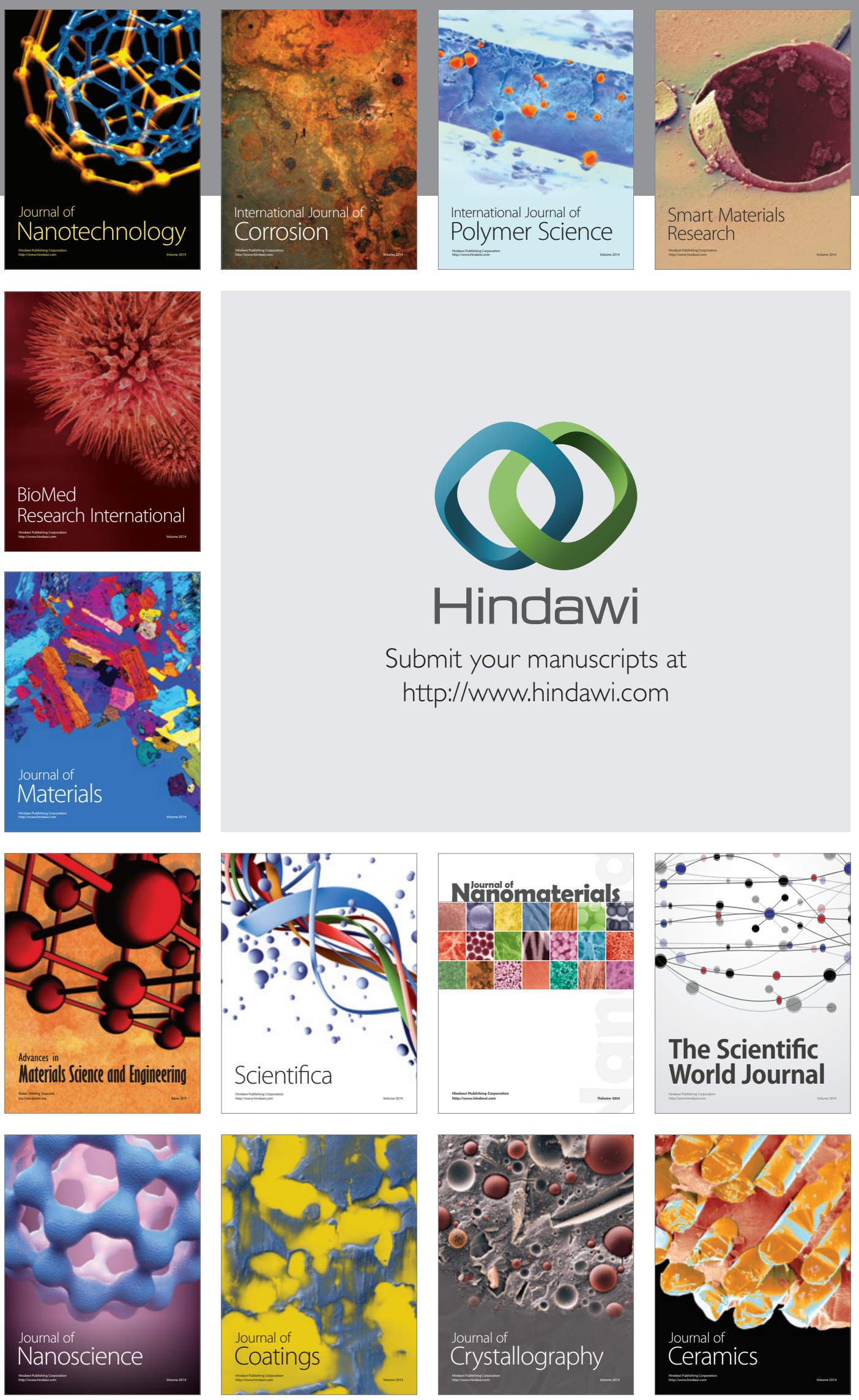

The Scientific World Journal

Submit your manuscripts at

http://www.hindawi.com

\section{World Journal}

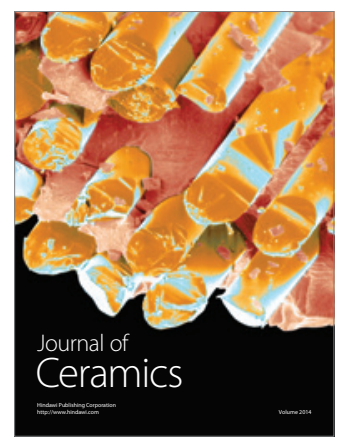

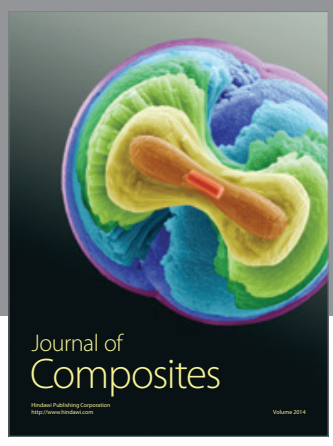
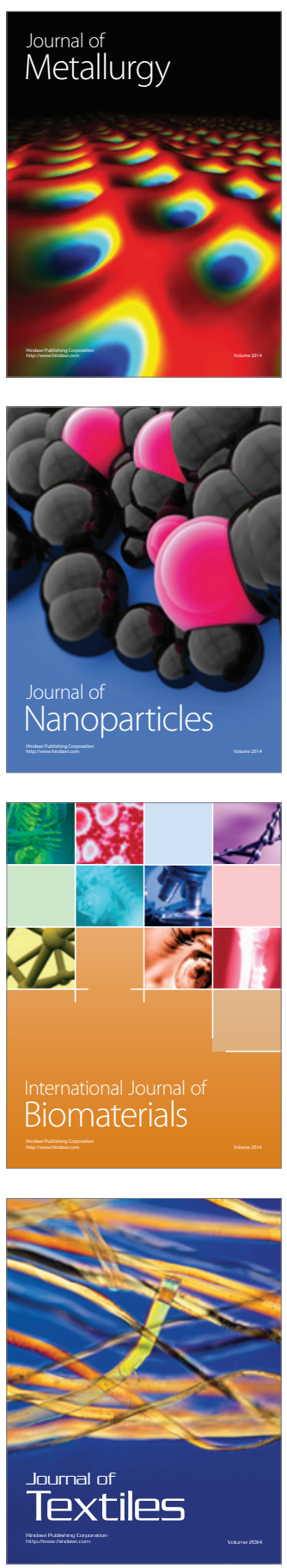